\title{
Investigations of a New Scheme for Wave Propagation
}

\author{
Doreen Fan* and Philip L. Roe ${ }^{\dagger}$ \\ Department of Aerospace Engineering, University of Michigan, Ann Arbor, MI 48109, USA.
}

\begin{abstract}
We conduct a comparison of the Active Flux method proposed by Eymann and Roe versus Discontinuous Galerkin with linear reconstruction (DG1). We find the Active Flux method capable of matching the accuracy of DG1 with a mesh spacing about three times greater, and capable of time steps about 2.5 times longer. On a given mesh, the Active Flux method can compute flows that should display circular symmetry with dramatically less scatter.
\end{abstract}

\section{Introduction}

This paper will investigate the performance of a method for solving wave propagation problems described at the 2011 Computational Fluid Dynamics Conference by Eymann and Roe. ${ }^{5}$ The method is capable of solving wave equations, such as those governing acoustic waves, electromagnetic phenomena, or elastic waves, on arbitrary unstructured grids. For linear problems it can be implemented to any order, and is exact on any grid whenever the initial data is a global polynomial of the same degree as the reconstruction. The order of accuracy is one order higher than this. The method is fully explicit. Experimentally, we find that it is stable up to the maximum time step that is physically allowed. For nonlinear problems, we have focused on thirdorder accuracy, because it is likely to be the best suited to aeronautical applications and also because in that case it is only necessary to allow for nonlinear effects by making a first-order correction to the wavespeed.

The method is being developed as one half of a project to solve the multidimensional Euler equations (and ultimately, of course, the Navier-Stokes equations). The approach to the Euler equations is based on a splitting of advective and acoustic terms, on account of their very different numerical requirements. Each of them is used as one ingredient in an Active Flux method. In this method the solution is represented by point values on the edges and vertices of finite volumes. and by the average solution within the volume. One timestep consists of using a reconstructed solution within each element to update the point values to second order. This stage takes the total update to be the sum of an advective update and an acoustic update. The timestep is completed by computing fluxes from the point values and then integrating around the boundary to obtain a third-order update of the cell average.

The acoustic part of the boundary update is the part that we treat here. It is based on Poisson's exact solution to the initial value problem for the scalar wave equation in terms of spherical means. In order to explain the idea, we begin by quickly recollecting van Leer's (1977) method for constructing one-dimensional linear advection schemes. Then we show how essentially the same idea can be applied to the multidimensional wave equation. We compare the cost and accuracy of our method with that of the Discontinuous Galerkin method using piecewise linear reconstruction. We think of this (DG1) method as being the method most competitive with ours.

\section{Method Description}

\section{A. "A new approach to numerical advection"}

This was the subtitle given ${ }^{8}$ by Bram van Leer to Part IV in his series "Toward the Ultimate Conservative Difference Scheme". Applied to the linear advection problem, $\partial_{t} u+a \partial_{x} u=0$ the idea is to begin by choosing some polynomial representation of the solution in each cell, and the parameters that will define

* Graduate Student, Member AIAA

${ }^{\dagger}$ Professor, Fellow, AIAA 

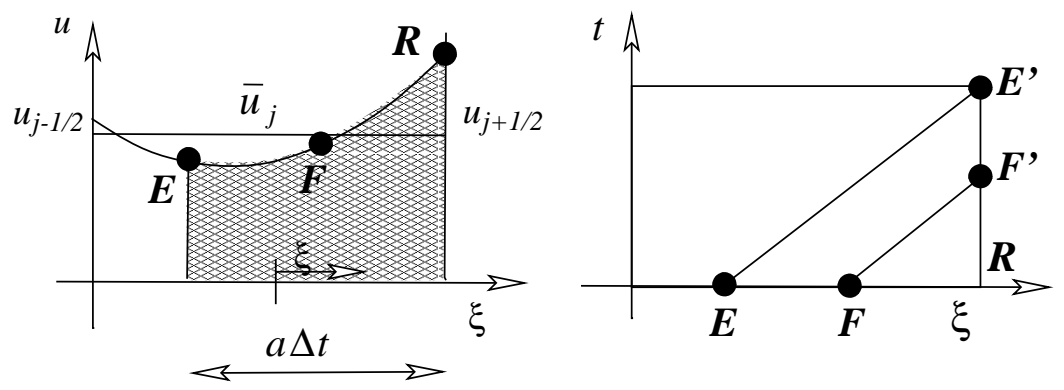

Figure 1. The construction of a third-order scheme for one-dimensional linear advection. At the beginning of the timestep we know the average value in the cell and the point values at the boundaries between the cells. Values at $E^{\prime}, F^{\prime}$ on the outflow boundary are found by tracing the characteristics backward to $E, F$.

it. For Scheme V the representation is quadratic, and degrees of freedom are the average value in each cell, $\bar{u}_{j}=\bar{u}_{M}$, and the point values $u_{j \pm 1 / 2}=u_{L, R}$ on each interface. The reconstruction obtained from this data is

$$
U(\xi)=\frac{(6 \xi+1)(2 \xi-1)}{4} u_{L}+\frac{3\left(1-4 \xi^{2}\right)}{2} \bar{u}_{M}+\frac{(6 \xi-1)(2 \xi+1)}{4} u_{R}
$$

where $\xi \in\left[-\frac{1}{2}, \frac{1}{2}\right]$ is a local coordinate within the cell. From this we wish to compute new values of the cell averages and the interface values. The interface value at $x_{j+1 / 2}$ after time $\Delta t$ follows at once from realizing that the linear advection problem is exactly solved by $u=U(x-a t)$, whence

$$
u_{j+1 / 2}(t+\Delta t)=U\left(\frac{1}{2}-\nu\right)=\nu(\nu-1) u_{L}+v(3-2 \nu) \bar{u}_{M}+(1-\nu)^{2} u_{R}
$$

The new value on the right edge will be

$$
u_{j+\frac{1}{2}}^{n+1}=\nu(3 \nu-2) u_{L}+6 \nu(1-\nu) \bar{u}_{M}+(1-3 \nu)(1-\nu) u_{R}
$$

To obtain the cell average, we first compute the flux through an interface, as

$$
\bar{u}_{j+\frac{1}{2}}^{n+\frac{1}{2}}=\frac{1}{\Delta t} \int_{x_{j+\frac{1}{2}-a \Delta t}}^{x+\frac{1}{2}} U(x) d x=\bar{u}_{M}+(1-n u)\left[(1-\nu) u_{R}-\bar{u}_{M}\right)+\nu\left(\bar{u}_{M}-u_{L}\right]
$$

and then updating the cell average simply by

$$
\bar{u}_{j}^{n+1}=\bar{u}_{j}^{n}-\nu\left(u_{j+\frac{1}{2}}^{n+\frac{1}{2}}-u_{j-\frac{1}{2}}^{n+\frac{1}{2}}\right)
$$

We have given this method in detail because the method that we propose follows exactly the same path. Both are based on the sequence: Reconstruction (obtaining the parabolic distribution from the initial data) Evolution (solving the reconstructed problem, in this case exactly) and Projection (restoring the solution to its original form of description). This has been a fruitful source of inspiration for algorithm design, and it turns on having available some simple problem that can be solved exactly, that is close enough in structure to the problems of real interest. Almost always this has been taken to be the linear scalar advection problem, which has become the numerical prototype for all hyperbolic problems.

Another equation for which the initial-value problem can be solved exactly is the multidimensional scalar wave equation, and this solution carries over to some related systems of first-order equations. This provides a basis for solving problems of multidimensional acoustics, and we now describe how the reconstructed problem can be solved, in that case also, by an exact method. The outcome will be a method that is truly based on the physics of multidimensional flow.

\section{B. The Poisson solution to the initial value problem}

Given a function $f(\mathbf{x})$ we define the spherical mean of that function $M_{r}\{f(\mathbf{x}, t)\}$ to be the mean value of $f$ over a sphere of radius $r$ centered on $\mathbf{x}$. The solution to the initial value problem for the scalar wave equation 


$$
\partial_{t t}^{2} \phi=c^{2} \nabla^{2} \phi
$$

is given in textbooks on partial differential equations ${ }^{4,9}$ as

$$
\phi(\mathbf{x}, t)=\partial_{t}\left(t M_{c t}\{\phi(\mathbf{x}, 0)\}\right)+c t M_{c t}\left\{\partial_{t} \phi(\mathbf{x}, 0)\right\}
$$

A method similar to ours and based on this formula has been proposed ${ }^{1,6}$ for the scalar wave equation. However, this is not the form of the wave equation that appears within the Euler equations. For one thing, it assumes irrotational flow.

This formula can nevertheless be adapted to solve the linear first-order system form. We are particularly interested here in solving the acoustic system

$$
\begin{array}{r}
\partial_{t} p+c \nabla \cdot \mathbf{v}=0 \\
\partial_{t} \mathbf{v}+c \nabla p=0
\end{array}
$$

The formula (6) applies directly to the pressure because that variable can easily be shown to obey the scalar wave equation (5), But the velocities do not obey (5) unless the flow is irrotational. A case where the flow is irrotational was presented in. ${ }^{5}$ At that time it was thought that the rotational case would need to be handled by a different formula, perhaps with extra terms, but there is however, another form of the Poisson formula that can be seen to apply to all variables. Manipulation allows (6) to be rewritten as

$$
\phi(\mathbf{x}, t)=\phi(\mathbf{x}, 0)+t M_{c t}\left\{\partial_{t} \phi(\mathbf{x}, 0)\right\}+\int_{0}^{c t} t M_{c t}\left\{\nabla^{2} \phi(\mathbf{x}, 0)\right\} d t
$$

If we assume for the moment that (9) does apply to the acoustic system, we can insert the values of the time derivatives to obtain

$$
\begin{aligned}
& p(\mathbf{x}, t)=p(\mathbf{x}, 0)-c t M_{c t}\{\nabla \cdot \mathbf{v}\}+\int_{0}^{c t} t M_{c t}\left\{\nabla^{2} p\right\} d t \\
& \mathbf{v}(\mathbf{x}, t)=\mathbf{v}(\mathbf{x}, 0)-c t M_{c t}\{\nabla p\}+\int_{0}^{c t} t M_{c t}\left\{\nabla^{2} \mathbf{v}\right\} d t
\end{aligned}
$$

Now it is easy to show that, if the velocities are divided into an irrotational component $\mathbf{v}_{I}$ and a rotational component $\mathbf{v}_{R}$, such that $\nabla \times \mathbf{v}_{I}=\nabla \cdot \mathbf{v}_{R}=0$, then

$$
\partial_{t t}^{2} \mathbf{v}_{I}=c^{2} \nabla^{2} \mathbf{v}_{I}, \quad \partial_{t} \mathbf{v}_{R}=0
$$

so that clearly (11) gives the irrotational velocities. Since the rotational velocities do not change with time, they are given correctly by the first term. They have no effect on the third term by their definition.

From a numerical analysis point of view, the solution $(10,11)$ is rather interesting. The first term is just the current solution, while the second and third terms are of order $\Delta t,(\Delta t)^{2}$, giving the appearance of a Lax-Wendroff formula. In fact a Lax-Wendroff finite difference method would result from evaluating each term pointwise, but the formula becomes exact if the terms are evaluated exactly.

Another interesting aspect of the exact solution $(10,11)$ is that changes in pressure are driven by the divergence of velocity, rather than by velocity gradients, as is implied by the use of methods based on onedimensional Riemann solvers, or other dimension-by-dimension approaches. We believe that this is significant for other aspects of algorithm design, such as limiting. ${ }^{7}$

Although the formula is derived directly in three dimensions, two-dimensional problems can be handled by considering data that depends only on $x, y$ and for which $w=0$, or only on $x$ with $v=w=0$. In the two-dimensional case, we have, using polar coordinates

$$
M_{R}\{u(x, y)\}=\frac{1}{2 \pi R} \int_{0}^{2 \pi} \int_{0}^{R} \frac{r u(x+r \cos \theta, y+r \sin \theta)}{\sqrt{R^{2}-r^{2}}} d r d \theta
$$

All of our numerical experiments are in two dimensions. The solution is then given by substituting this revised definition of $M_{R}$ into $(10,11)$. 
In one dimension the spherical mean is very simple;

$$
M_{R}\{u(x)\}=\frac{1}{2 R} \int_{-R}^{R} u(x) d x
$$

and the solution reduces to to the method of characteristics

$$
\begin{aligned}
& p(x, t)=\frac{1}{2}(p(R, 0)+p(-R, 0))-\frac{R}{2}(u(R, 0)-u(-R, 0)) \\
& u(x, t)=\frac{1}{2}(u(R, 0)+u(-R, 0))-\frac{R}{2}(p(R, 0)-p(-R, 0))
\end{aligned}
$$

\section{Numerical Method}

In two dimensions, we suppose an arbitrary triangulation of the plane. A quadratic interpolant over each element is defined by introducing Lagrange points at the vertices and edge midpoints. The standard Lagrange basis functions for these degrees of freedom are given in barycentric coordinates by $4 s_{i} s_{j},\left(s_{i}-1\right)\left(2 s_{i}-1\right)$. We introduce an additional degree of freedom in the form of a bubble function $27 s_{1} s_{2} s_{3}$. To advance by one timestep we begin by considering discs of radius $c \Delta t$ centered on each boundary point, as in Figure 2 . The update to each such point consists of integrating (13) over each of these circles, one segment at a time.

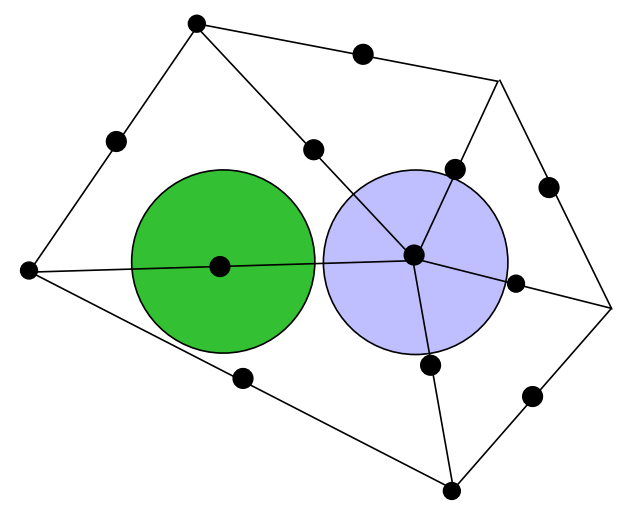

Figure 2. Each point value is updated by integrating over the disc of radius $c \Delta t$ surrounding it. For stability, a necessary condition is that no disc escapes from any the elements that it belongs to.

When transferred to local coordinates the integrals that have to be computed are all of the form

$$
I_{p}=\int_{\theta_{1}}^{\theta_{2}} \int_{0}^{c t} \frac{r^{p} \sin ^{p-1} d r d \theta}{\sqrt{c^{2} t^{2}-r^{2}}}
$$

where $p$ takes all integer values less than or equal to the order of the method plus one. For the third-order method we need

$$
\begin{aligned}
& I_{1}=a t\left(\theta_{2}-\theta_{1}\right) \\
& I_{2}=\frac{\pi a^{2} t^{2}}{4}\left(\cos \theta_{2}-\cos \theta_{1}\right), \\
& I_{3}=\frac{a^{3} t^{3}}{3}\left(\theta_{2}-\theta_{1}+\cos \theta_{1} \sin \theta_{1}-\cos \theta_{2} \sin \theta_{2}\right) \\
& I_{4}=\frac{\pi a^{4} t^{4}}{16}\left(\cos \theta_{1}\left(2+\cos ^{2} \theta_{1}\right)-\cos \theta_{2}\left(2+\cos ^{2} \theta_{2}\right)\right)
\end{aligned}
$$

The last integral is needed to calculate the effect of the bubble function, the need for which is shown below. Since all of these integrals have the form $f(t)\left(g\left(\theta_{1}\right)-g\left(\theta_{2}\right)\right)$ and each $g(\theta)$ appears in the evaluation of six integrals, the values of $g(\theta)$ can advantageously be precomputed. The integrals required in the threedimensional case have been given by Beckmann, Mhaskar, and Prestin, ${ }^{2}$ in a paper on the integration of scattered geophysical data. 


\section{Remarks}

We will frequently make comparison with the linear Discontinuous Galerkin method (DG1) and make some remarks here that are relevant to that comparison.

1. For the AF method in two dimensions there are locally seven degrees of freedom to each element. There are three degrees of freedom associated with the vertices, but each of these is shared with about six neighbors, and so they contribute 0.5 to the global storage requirement. There are three degrees of freedom associated with edges, each shared between two elements; they contribute 1.5 to the global requirement. Adding in the bubble function gives three global degrees of freedom per element, which is the same as the standard form of DG1. In three dimensions we have one bubble function, plus six edge values each shared by about six neighbors on average, and four vertex values, each shared between about twenty neighbors, for a total of 2.20. A conventional DG method uses four.

2. The physical requirement for stability is that the convex hull of the point values used to update a given point value must include the physical domain of dependence. Geometrically, this means that no disc can cross over any element to which it belongs. We define a local Courant number for each point, which equals unity when this condition is first violated. Experimentally, we find that our code runs stably provided all local Courant numbers remain less than unity. Our method is therefore maximally stable. When a DG1 code is run on the same grids, the maximum time step is only about $40 \%$ of this. This corresponds to the factor of 0.406 that is found in one space dimension. ${ }^{3}$

\section{Nonlinear problems}

We have made numerical experiments on nonlinear problems of the form

$$
\begin{aligned}
\partial_{t} \rho+\nabla \cdot \mathbf{v} & =0 \\
\partial_{t} \mathbf{v}+\nabla p(\rho) & =0
\end{aligned}
$$

For a third-order scheme the fluxes need only be second-order accurate. Since the linearized fluxes are firstorder accurate, only the leading nonlinear correction is needed. So the point values are evolved according to the linear formulas, except that the disc around each point is assigned a radius proportional to the local sound speed, given by $a^{2}=p^{\prime}(\rho)$. Otherwise the integrals are unchanged. This is rather similar to using a locally linearized Riemann solver.

\section{Results}

All the results presented here are for the acoustic wave equation (18) either for the linear case $p=\rho$ or the nonlinear case $p=\rho^{\gamma}$.

\section{A one-dimensional test}

It was shown above that the Poisson formulas reduce analytically to the standard method of characteristics in one dimension. The numerical methods in one and two dimensions will not exactly coincide because they employ different degrees of freedom. To compare them we considered a one-dimensional acoustic problem with initial data consisting of a square pressure pulse. We solved this, either by applying the one-dimension method of characteristics using the original Scheme V, or by applying the two-dimension method described above. The results appear in Figure 3. Although the results are not identical, they are so close that it cannot be said that either one is better. In both cases the oscillations at the discontinuity are much smaller and more contained than they would be from a second-order method.

\section{A Gaussian pressure pulse}

We have made numerous calculations of a problem where the fluid is initially at rest and

$$
p(x, y, 0)=1+k e^{-50\left(x^{2}+y^{2}\right)}
$$

We have studied a linear problem with $p(\rho)=\rho$ and $k=1$, and a nonlinear problem with $p(\rho)=\rho^{1.4}$ and $k=2$. 

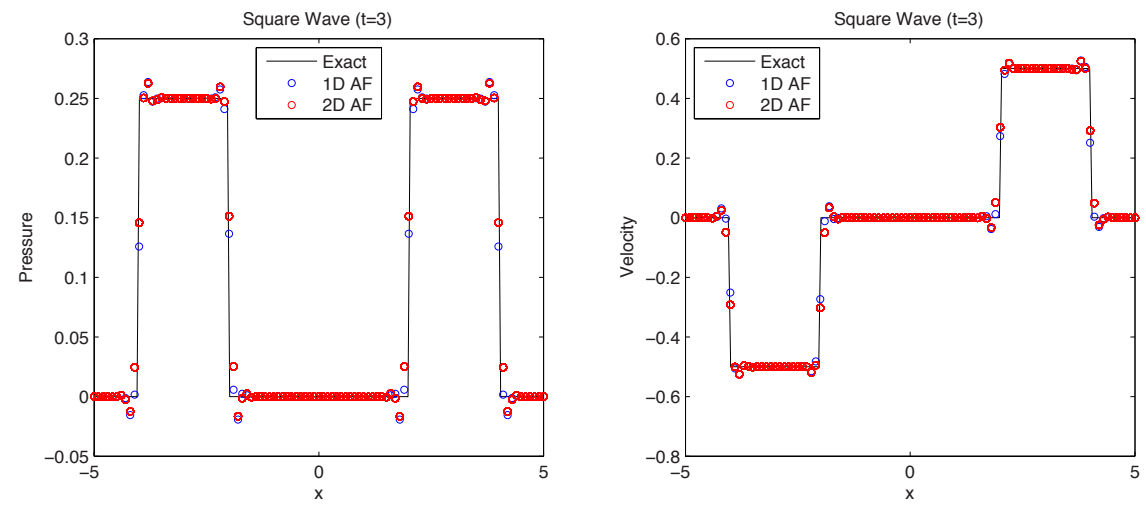

Figure 3. An initial square pressure pulse gives rise to two pulses moving in opposite directions.

Fig 4 shows the pressure and velocity magnitude at time $t=1$ in the linear problem. The red symbols, obtained from the DG1 method, exhibit considerably more scatter than the blue symbols, obtained from AF. The scatter is greater in the velocity distribution than it is in the pressure and, of course, much more noticeable in the places where the solution changes rapidly. Close-ups of these locations are provided in Figure 5. We surmise that the greater isotropy of the AF results comes from the mesh invariance implicit in the Poisson equation.
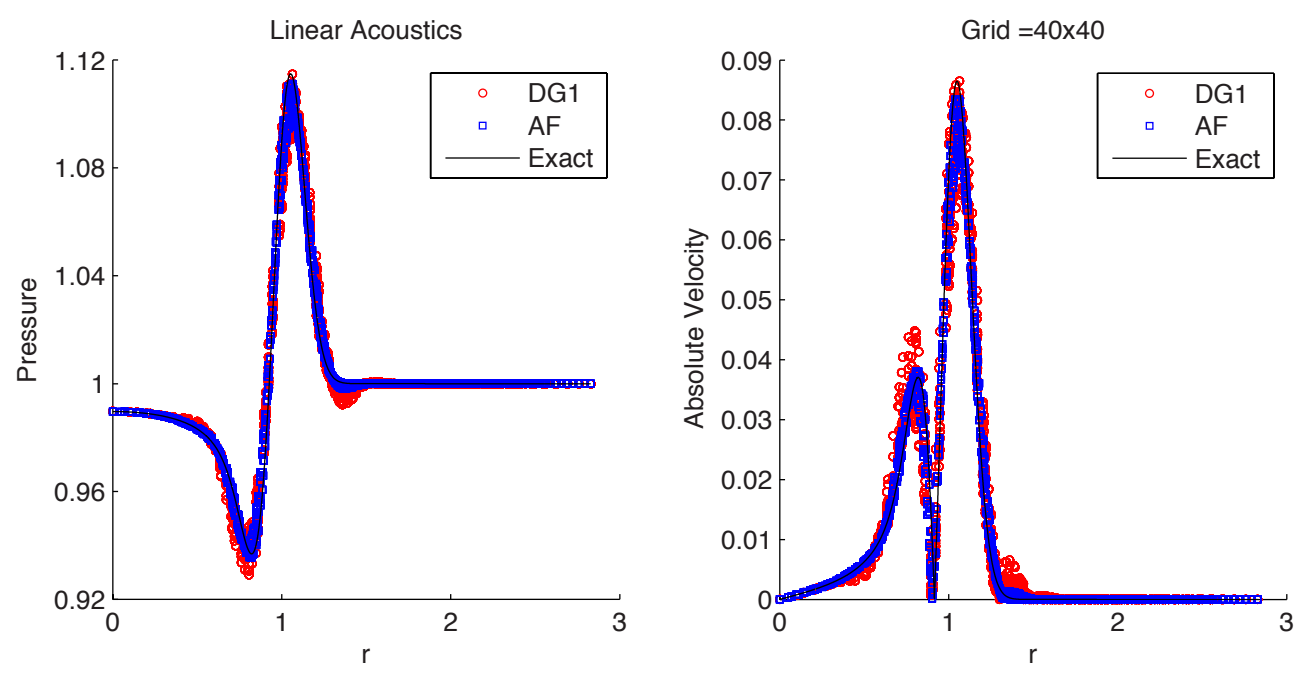

Figure 4. Pressure (left) and velocity magnitude (right) at $t=1$ for the pressure pulse problem. All of the nodal values are plotted against the radius of the node.

Mesh convergence was examined by computing a scalar output consisting of a certain weighted integral of the pressure distribution. The error in this integral was determined by comparison with a reference solution computed using the fifth-order DG2 method on a $160 \times 160$ grid. The results are shown in Figure 6 . We observe third-order convergence for both methods ${ }^{\mathrm{a}}$; in fact the AF method seems to be converging slightly faster than this. Over the range of mesh sizes used, the AF method accepted a roughly three times larger mesh spacing ${ }^{\mathrm{b}}$ than DG1. This would imply taking nine times fewer mesh points, and $3 / 0.4=7.5$ times fewer time steps. This gives the advantage to $\mathrm{AF}$ as $9 \times 7.5=67.5$ fewer time advances to reach a given final time. We are not able to give an accurate evaluation of the relative $\mathrm{CPU}$ times, because both codes were research codes that had not been designed for efficiency.

We also ran a nonlinear version of this problem and the results can be seen in Figure 7 . In this case the

\footnotetext{
${ }^{a}$ Initially we were only able to observe second-order convergence for DG1, and the third-order result was only obtained after very carefully choosing the initialization of the data. There were no such problems with AF.

${ }^{\mathrm{b}}$ This is consistent with previous observations in one dimension.
} 

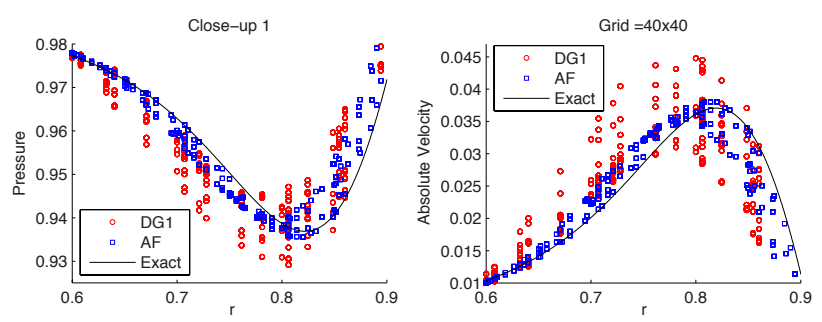

Figure 5. Close-up plots of the results for $0.6 \leq r \leq 0.9$

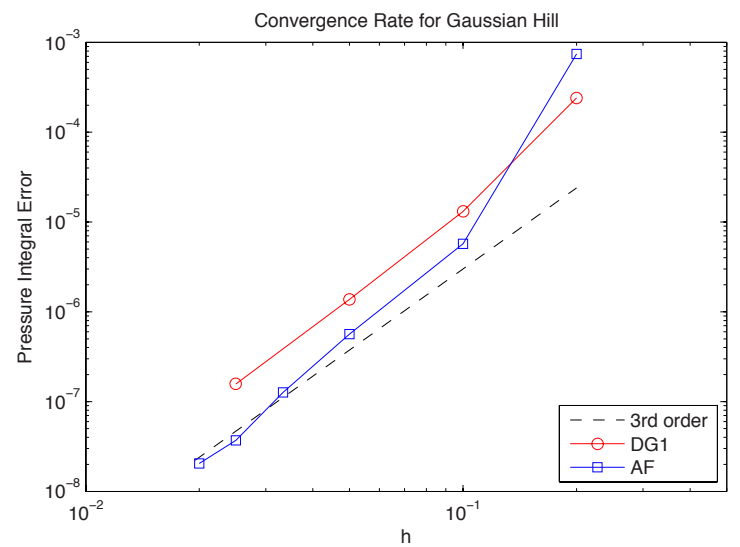

Figure 6. Convergence plot for each method applied to the linear Gaussian pulse problem.

compression wave steepens as it moves out and forms a shockwave. Figure 7 compares the AF and DG1 results just after the shock has formed on an $80 \times 80$ grid. There is increased scatter in the results of both methods. This can be explained by observing that, with the chosen grid, the grid resolution is different in different directions, which means that some regions of the shock are better resolved than others. The peak overdensity from the AF method ranges from about $11 \%$ to $15 \%$ as compared with about $9 \%$ to $13 \%$ from DG1. That the value of $15 \%$ is correct is confirmed by both methods on an 160x160 grid. We again show a closeup in Fig 8. Notice that the expanded horizontal axis has made the shock appear smeared, but the AF solution is more crisply defined (by a factor of about two) than the solution from DG1.

\section{Double sine wave}

The linear version of (18) has an analytical solution

$$
\begin{aligned}
p(x, y, t) & =\frac{1}{c} \cos 2 \pi c t[\sin 2 \pi x+\sin 2 \pi y] \\
u(x, y, t) & =\frac{1}{c} \sin 2 \pi c t \cos 2 \pi x \\
v(x, y, t) & =\frac{1}{c} \sin 2 \pi c t \cos 2 \pi y
\end{aligned}
$$

For our experiments we took $c=1$ and calculated from $t=0$ to $t=1.0$, by which time the original data should repeat itself. The outcome of this experiment is shown in Figure 9. This time there is a large difference between the methods because although they are both third-order in pressure, the DG1 results are only second-ordr in the velocities.

We also carried out tests on this problem, investigating the ability of each method to operate with a severely distorted "torture" grid. The grid is shown in Figure 10. The square domain was divided into four quadrants, three of which were left as they were, but the North-East quadrant was deliberately polluted with small, badly formed elements. We measured the error by the absolute value of the difference between the numerical and exact values of the cell average. Both methods gave errors in the pressure (left-hand pictures) 

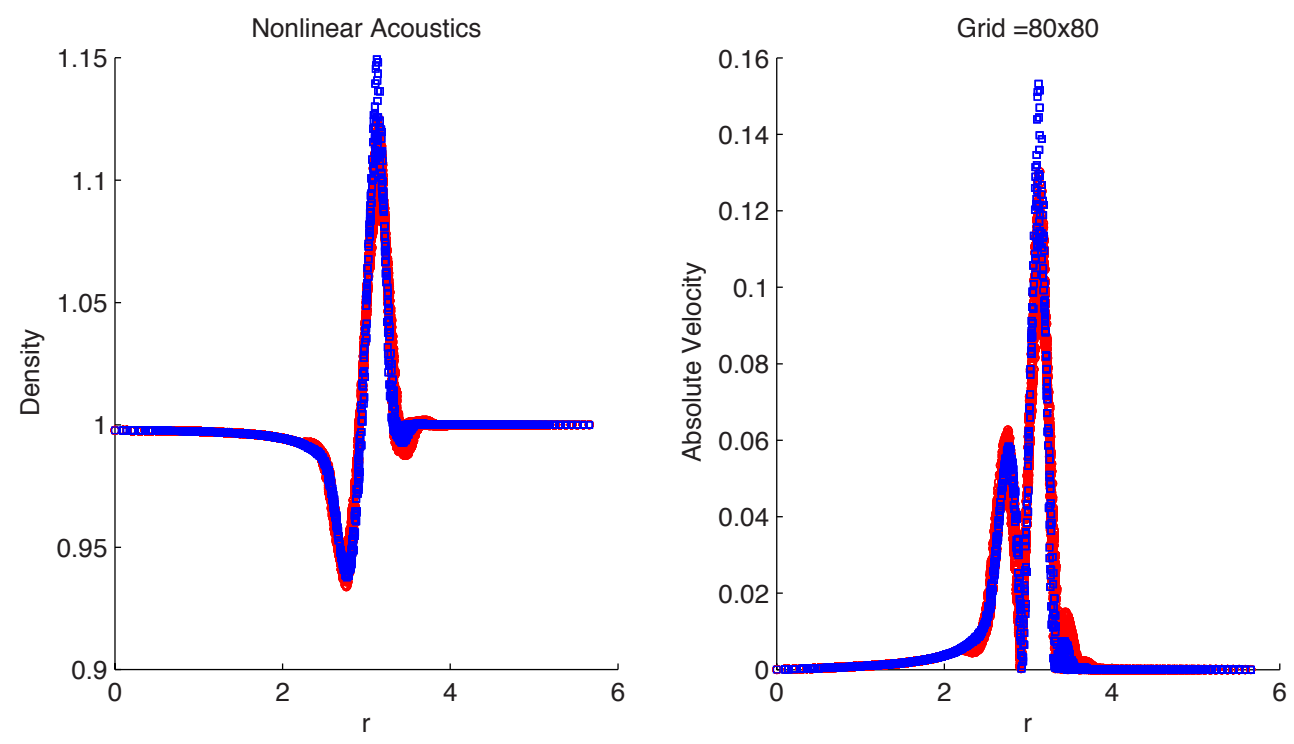

Figure 7. Density (left) and velocity magnitude (right) at $t=3.0$ for the nonlinear pressure pulse problem. All of the nodal values are plotted against the radius of the node.
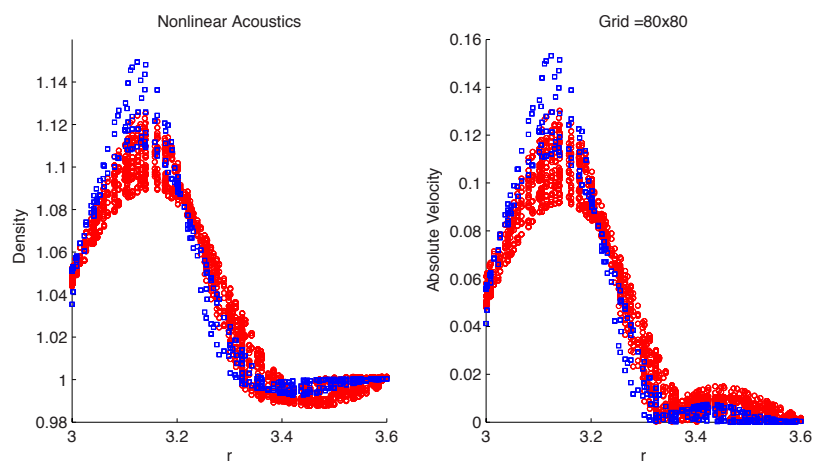

Figure 8. Closeup plots of density (left) and velocity magnitude (right) at $t=3.0$ for the nonlinear pressure pulse problem. The AF method demonstrates a steeper shock and a better prediction of the peak value.

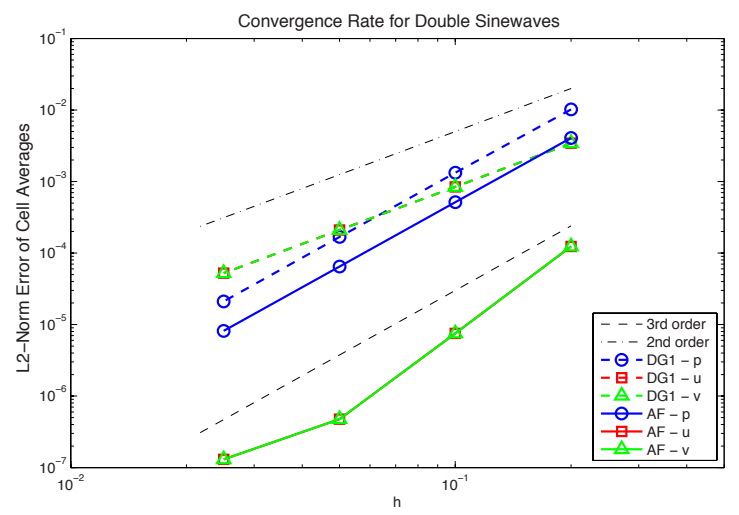

Figure 9. Convergence of the two methods for the double sinewave problem. 


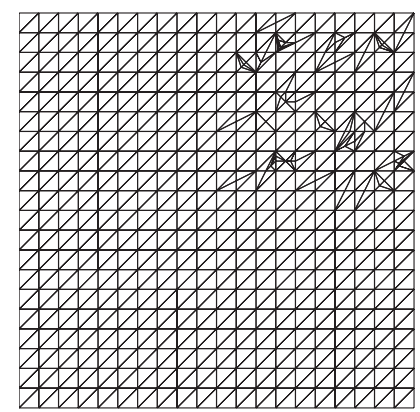

Figure 10. The distorted grid used to test tolerance of poor meshes.

that were surprisingly well-behaved; the errors from the AF method were a little smaller. The errors in speed were fairly similar in speed for DG1, but reduced by a factor of about four for AF.
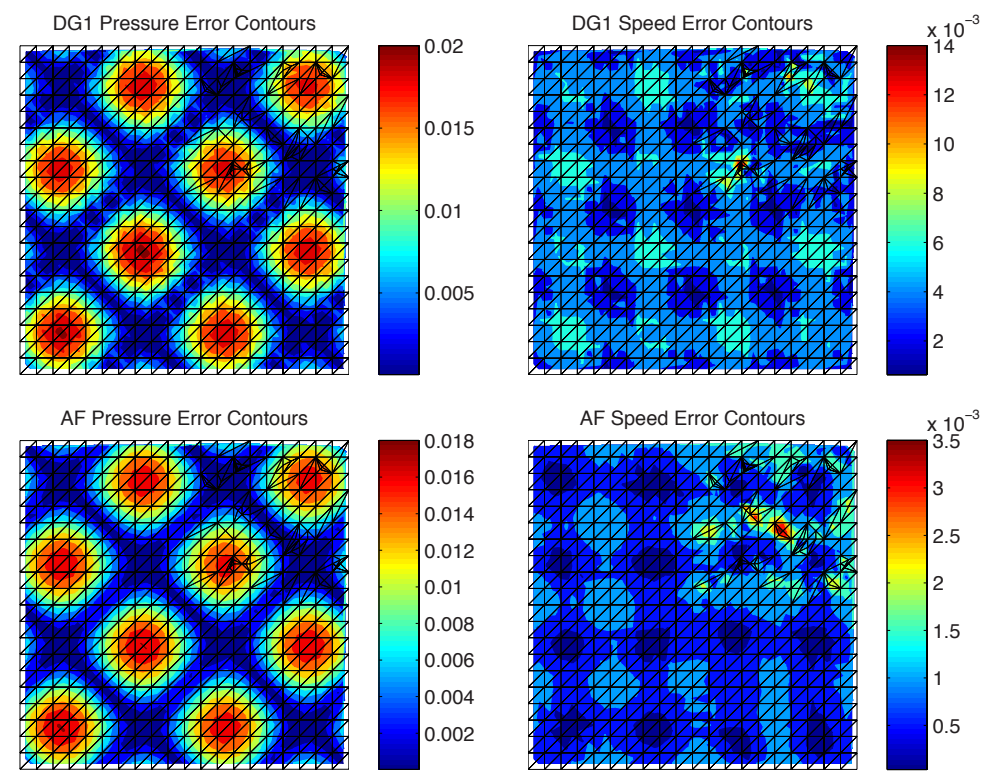

Figure 11. Contours of error for the double sine waves computed on the "torture test" grid. At left, pressure and at right, speed. At top, DG1 and at bottom Active flux.

\section{Conclusions}

The purpose of this paper is to evaluate a new method (Active Flux) of solving wave equations numerically, and to compare it with the Discontinuous Galerkin method using linear reconstruction and known as DG1. Over a variety of tests, AF was found able to match the accuracy of DG1, with a mesh spacing about three times larger. The Courant number could be 2.5 times larger. Both of these measures are similar to what is found in one dimension and if such comparisons continue to hold in three dimensions, the AF method would be superior by a factor of about two hundred, made up of three space dimensions $\left(3^{3}\right)$ times one time dimension (3) times the greater stability range (2.5). This is of course a naive preliminary assessment, which neglects, among other important considerations, the expense of each time advance. We hope to report on this aspect soon. Additionally, the AF method provided better-resolved shocks, converged with third-order accuracy on every test, and suffered less from the effects of severe mesh distortion.

On the other hand, it is not certain that either method will retain full third-order accuracy when extended to the full Euler equations. Doubt may be entertained with regard to DG1, which relies for its third-order accuracy on superconvergence, an effect that is still little understood and is rarely observed in complex 
situations. If this should fail, then the next available Discontinuous Galerkin method, DG2, is considerably more expensive. AF relies on the validity of superposing advective and acoustic effects, something so far demonstrated only for linear problems. This is current work.

\section{Acknowledgments}

We thank Professor Chris Fidkowski and Kyle Ding for running the comparisons with DG1.

\section{References}

\footnotetext{
${ }^{1}$ Alpert, B., Greengard, L., Hagstrom, T., An Integral Evolution Formula for the Wave Equation, J. Comput. Phys. 162,pp. 536543, 2000.

${ }^{2}$ Beckmann, J., Mhaskar, H.N., Prestin, J., Quadrature formulas for integration of multivariate trigonometric polynomials on spherical triangles, Int. J. Geomath., 3, pp 119-138, 2012.

${ }^{3}$ Cockburn, B., Shu, C-W., RungeKutta Discontinuous Galerkin Methods for Convection-Dominated Problems, J. Sci. Comput., 16(3), pp 173-261, 2001.

${ }^{4}$ Courant, R., Hilbert, D., Methods of mathematical physics, vol 2, Interscience, 1962.

${ }^{5}$ Eymann. T.A., Roe, P. L., Active Flux Schemes for systems. AIAA paper 2011-3840, AIAA CFD Conference, Hawaii, 2011.

${ }^{6} \mathrm{Li}$, J-R., Greengard, L., High order marching schemes for the wave equation in complex geometry, J. Comput. Phys. 198,pp. 295-309, 2004.

${ }^{7}$ Roe, P. L., Lung, T.B., Maeng, J., New approaches to limiting, AIAA paper, this CFD Conference, Dallas, 2015.

${ }^{8}$ van Leer, B., Toward the ultimate conservative differencing scheme, IV: a new approach to linear convection. J. Comp. Phys., 23, pp 276-299, 1977.

${ }^{9}$ Whitham, G. B., Linear and nonlinear waves, Wiley-Interscience, 1974.
} 